\title{
Hydrogen Rich Water Attenuates Renal Injury and Fibrosis by Regulation Transforming Growth Factor- $\beta$ Induced Sirt1
}

\author{
Zhaoyu Xing, ${ }^{a, \#}$ Wanma Pan, ${ }^{b, \#}$ Jing Zhang, ${ }^{b}$ Xianlin Xu, ${ }^{a}$ Xuemei Zhang, ${ }^{b}$ Xiaozhou He, ${ }^{*, a}$ and \\ Min Fan*,a \\ a Department of Urology, The Third Affiliated Hospital of Soochow University; Changzhou 213000, Jiangsu Province, \\ China: and ${ }^{b}$ Department of Pharmacology, School of Pharmacy, Fudan University; Shanghai 201203, China. \\ Received October 25, 2016; accepted January 24, 2017
}

\begin{abstract}
The current research was designed to study the role of hydrogen in renal fibrosis and the renal epithelial to mesenchymal transition (EMT) induced by transforming growth factor- $\beta 1$ (TGF- $\beta 1$ ). Hydrogen rich water (HW) was used to treat animal and cell models. Unilateral ureteral obstruction (UUO) was performed on Balb/c mice to create a model of renal fibrosis. Human kidney proximal tubular epithelial cells (HK-2 cells) were treated with TGF- $\beta 1$ for $36 \mathrm{~h}$ to induce EMT. Serum creatinine (Scr) and blood urea nitrogen (BUN) were measured to test renal function, in addition, kidney histology and immunohistochemical staining of alpha-smooth muscle actin ( $\alpha$-SMA) positive cells was performed to examine the morphological changes. The treatment with UUO induced a robust fibrosis of renal interstitium, shrink of glomerulus and partial fracture of basement membrane. Renal function was also impaired in the experimental group with UUO, with an increase of Scr and BUN in serum. After that, Western-blot was performed to examine the expression of $\alpha$-SMA, fibronectin, E-cadherin, Smad 2 and Sirtuin-1 (Sirt1). The treatment with HW attenuated the development of fibrosis and deterioration of renal function in UUO model. In HK-2 cells, the pretreatment of HW abolished EMT induced by TGF- $\beta 1$. The down-regulation the expression of Sirt1 induced by TGF- $\beta 1$ which was dampened by the treatment with HW. Sirtinol, a Sirt1 inhibitor, reversed the effect of HW on EMT induced by TGF- $\beta 1$. HW can inhibit the development of fibrosis in kidney and prevents HK-2 cells from undergoing EMT which is mediated through Sirt1, a downstream molecule of TGF- $\beta 1$.
\end{abstract}

Key words hydrogen rich water; unilateral ureteral obstruction; transforming growth factor- $\beta 1$; Sirtuin-1

We have witnessed the benefits on the treatment of renal disease from the new technology and therapy in modern medicine in the last decades. However, renal transplantation remains the ultimate choice for patients with end-stage renal disease (ESRD). ${ }^{1,2)}$ Vascular anastomosis and immunosuppressor improve short-term survival of the allografts significantly after renal transplantation, but long-term survival is not satisfying. Chronic allograft nephropathy (CAN) is still the leading cause of allograft failure. ${ }^{2)}$ However, CAN could hardly be detected in the early-stage of allograft failure as urine protein and serum creatinine (Scr) are lacking of the specificity and sensibility to diagnose CAN, whose major pathological characteristic is renal fibrosis.

Up to now, hydrogen has been gaining attention for its protective effects in kidney. Protective effects with hydrogen rich water (HW) on kidney have been shown in rats with spontaneous hypertension, ${ }^{3)}$ metabolic syndrome in SHR.Cg-Leprcp/ NDmcr rat kidney, ${ }^{4)}$ renal ischemia-reperfusion injury, ${ }^{5)}$ nephrotoxicity induced by ferric nitrilotriacetate, ${ }^{6}$ gentamicin ${ }^{7)}$ or cisplatin, ${ }^{8)}$ etc. It has also been documented that HW improve metamorphosis and nephrotoxicity induced by reactive oxygen species (ROS) triggered by cisplatin. ${ }^{9}$ However, it remains unknown, how HW protect kidney from fibrosis. Smad2 and smad3 regulate the transcription of downstream fibrosis-related target genes, ${ }^{10,11)}$ which are considered to play a key role in the process of CAN.

Recently, several studies from different groups have shown the significant effect of Sirtl on anti-fibrosis in Diabetic Ne-

\footnotetext{
"These authors contributed equally to this work.

phropathy (DN) and Acute Kidney Injury (AKI). ${ }^{12-15)}$ Silent information regulator (Sir) is a family of genes which participates in the regulation of lifespan. ${ }^{16)}$ Sirtuins (Sirt), mammalian homologs of Sir2, is a family of nicotinamide adenine dinucleotide-dependent deacetylases. ${ }^{17)} \mathrm{Up}$ to date, seven sirtuins (Sirt1-7) have been found in mammals, localizing in different subcellular compartments such as the nucleus (Sirt1, 2, 6, 7), cytoplasm (Sirt1, Sirt2), mitochondria (Sirt3, 4, 5) which have exert functions in many physiological and pathological processes such as cancer, diabetes, neurological diseases and regulation of lifespan. ${ }^{18-20)}$ As we all know, increasing evidences have revealed the significant effect of Sirtl on antifibrosis in DN and AKI. ${ }^{12-15)}$

Hydrogen exists virtually everywhere in nature which is found in almost every chemical compound. In this study, we hypothesized that HW is able to alleviate renal fibrosis induced by transforming growth factor- $\beta 1$ (TGF- $\beta 1$ ) by impacting Sirtl. And the aim of this study is to find out a noninvasive method for treating CAN, which may also be a novel target of the treatment for CAN.

\section{MATERIALS AND METHODS}

Reagents TGF- $\beta 1$ was purchased from R\&D Systems (Minneapolis, MN, U.S.A.). Sirtinol from Sigma (Saint Louis, MO, U.S.A.) was reconstituted with dimethyl sulfoxide (DMSO) to make $10 \mathrm{~mm}$ of stock solution according to the product instruction. Antibodies against alpha-smooth muscle actin ( $\alpha$-SMA) and E-cadherin were bought from Epitomics (Burlingame, CA, U.S.A.). Antibody against fibronectin was 
obtained from Sigma. Antibodies against Sirt1 and Smad2 were brought from Cell Signaling Technology (Danvers, MA, U.S.A.). Antibody against glyceraldehyde-3-phosphate dehydrogenase (GAPDH) was bought from Santa Cruz Biotechnology (Dallas, Texas, U.S.A.). BCA Protein Assay Kit was bought from Shanghai Biocolor BioScience \& Technology Company (Shanghai, China).

Preparation of HW HW was produced as that in previous studies. ${ }^{21)}$ In short, purified hydrogen gas was dissolved into water under the pressure of $0.6 \mathrm{MPa}$ for $2 \mathrm{~h}$ and stored in an aluminum bag without dead volume at $4^{\circ} \mathrm{C}$.

Cell Culture and Hydrogen Treatment HK-2 cells were purchased from American Type Culture Collection (Rockville, MD, U.S.A.) and they were cultured in RPMI 1640 supplemented with $2000 \mathrm{mg} / \mathrm{L} \mathrm{NaHCO} 3,10 \%$ fetal bovine serum (FBS) (GIBCO BRL, Co., Ltd., U.S.A.), $100 \mathrm{U} / \mathrm{mL}$ penicillin and $100 \mu \mathrm{g} / \mathrm{mL}$ streptomycin in an incubator with $5 \% \mathrm{CO}_{2}$ at $37^{\circ} \mathrm{C}$. When the cells reached a confluence of $90 \%$, they were trypsinized and subcultured by a split ratio of one third in new culture flasks. For the treatment with hydrogen, HK-2 cells were seeded into culture dishes and cultured for $4 \mathrm{~h}$. Then, some dishes of medium were replaced to medium containing hydrogen medium. Before we stimulated with TGF- $\beta 1$, cells were incubated at $37^{\circ} \mathrm{C}$ for $12 \mathrm{~h}$ in culture medium containing molecular hydrogen or in normal culture medium. Cell culture media were replaced three times in the morning, at noon and in the evening every day.

Western Blot Analysis HK-2 cells were harvested in RIPA lysis buffer after washing three times with ice-cold phosphate buffered saline (PBS). The supernant was collected after centrifugation at $10000 \times \mathbf{g}$ for $15 \mathrm{~min}$ at $4^{\circ} \mathrm{C}$ and used later for Western blot as cell lysates. The protein concentration of cell lysates was detected by BCA Protein Assay. After being heated for $10 \mathrm{~min}$ at $95^{\circ} \mathrm{C}, 20 \mu \mathrm{g}$ of cell lysates were loaded separated by $10 \%$ sodium dodecyl sulfate polyacrylamide gels (SDS-PAGE) and transferred onto polyvinylidene difluoride (PVDF) membranes. The membranes were blocked with 5\% milk in Tris-buffered saline with $0.1 \%$ Tween-20 (TBST) for $2 \mathrm{~h}$ in the environment of room temperature, followed by an incubation with primary antibodies at $4{ }^{\circ} \mathrm{C}$ overnight. The secondary antibody conjugated with horseradish peroxidase (HRP) was incubated with the membranes for $2 \mathrm{~h}$ in the environment of room temperature before the enhanced chemiluminescent (ECL) substrate was applied to visualize the target protein blots. GAPDH was used as the loading controls.

Animals and Unilateral Ureteral Obstruction (UUO) Model Animal experiments were carried out under the rules and regulations of the animal care and use committee at Fudan University (Shanghai, China). Adult male Balb/c mice, weighing $25 \pm 3 \mathrm{~g}$, were used in this study. Mice were housed under a light or dark cycle per $12 \mathrm{~h}$ and maintained with free access to food and water.

A total of 24 mice were used and they were divided randomly into four groups (6 animals for each group): control group, sham group, UUO group, and UUO+HW group. To create the UUO model, animals were anesthetized with chloral hydrate and then UUO was performed under aseptic conditions. The left kidney and ureter were exposed through a midline incision in abdominal cavity, and the ureter was occluded with two 5-0 sterile sutures at two points. The latter part was ligated permanently to the uretero-pelvic junction. Animals in sham group underwent similar surgical procedures with no ligation of the left ureter. The control group, sham group and UUO group were supplied with normal water, while the HW+UUO group was supplied with HW. We supplied enough normal water or $\mathrm{HW}$ to the mice twice per day according to their groups for $3 \mathrm{~d}$ before surgery and thereafter for $10 \mathrm{~d}$. The average volume of consumption of normal water or HW was $4 \mathrm{~mL}$ per day for each mouse. After $10 \mathrm{~d}$, the blood and left kidneys of animals were collected for the biochemical analysis and immunohistologic examinations.

Immunohistochemical Staining The tissues were fixed in $4 \%$ paraformaldehyde, embedded in paraffin, and transversely sliced at a thickness of $4 \mu \mathrm{m}$. Then, the general histology was determined by hematoxylin and eosin staining (H\&E staining) and Masson staining respectively.

Renal sections were deparaffinized in xylene, rehydrated in graded ethanol and then they were treated with $0.3 \%$ hydrogen peroxide for $30 \mathrm{~min}$ to quench endogenous peroxidase activity for immunohistological staining. Antigen was retrieved by heating the sections in citrate buffer in a microwave oven for $5 \mathrm{~min}$. After blocking non-specific binding in $1 \%$ of bovine serum albumin (BSA) for $10 \mathrm{~min}$, sections were incubated with primary antibodies of $\alpha$-SMA for $2 \mathrm{~h}$. Afterward, the sections were incubated with secondary antibodies for $1 \mathrm{~h}$ after washing away primary antibodies and being conjugated with HRP-streptavidin for $30 \mathrm{~min}$. Finally, $\alpha$-SMA positive cells were visualized with chromogen diaminobenzidine (DAB). At last, the slides were counterstained with hematoxylin.

Statistical Analysis All data were expressed as mean \pm strandard error of the mean (S.E.M.). Statistical differences were performed by one-way ANOVA followed by Tukey's multiple comparison tests. A $p<0.05$ was considered statistically significant.

\section{RESULTS}

The Treatment with HW Rescued Renal Function in UUO Model UUO is a model used to investigate mechanisms of renal fibrosis. ${ }^{22,23)}$ With ureter ligation in the left kidney, BUN and Scr increased significantly in UUO group compared with normal group and sham group (Table 1, $p<0.05$ ), indicating renal dysfunction appeared. After the treatment with HW, the increase in BUN and Scr was dampened (UUO+HW vs. UUO, $p>0.05$ ) and rescued renal function partially.

The Treatment with HW Inhibited the Development of Interstitial Fibrosis in the Kidney Histologic examination of kidney showed UUO caused severe interstitial fibrosis, with

Table 1. The Treatment with HW Rescued Renal Function in UUO Model

\begin{tabular}{lcl}
\hline \hline \multirow{2}{*}{ Group } & \multicolumn{2}{c}{$\bar{x} \pm$ S.D. } \\
\cline { 2 - 3 } & BUN $(\mathrm{mmol} / \mathrm{L})$ & Scr $(\mu \mathrm{mol} / \mathrm{L})$ \\
\hline Normal & $7.747 \pm 1.379$ & $28.261 \pm 5.741$ \\
Sham & $8.3176 \pm 1.340$ & $30.822 \pm 6.423$ \\
UUO & $11.231 \pm 2.504^{\mathrm{a}}$ & $40.015 \pm 7.445^{\mathrm{b}}$ \\
UUO+HW & $9.022 \pm 1.611$ & $32.063 \pm 7.577$ \\
\hline
\end{tabular}

BUN: ${ }^{\mathrm{a}} p<0.05$ vs. Sham group, Scr: ${ }^{\mathrm{b}} p<0.05$ vs. Sham group. 

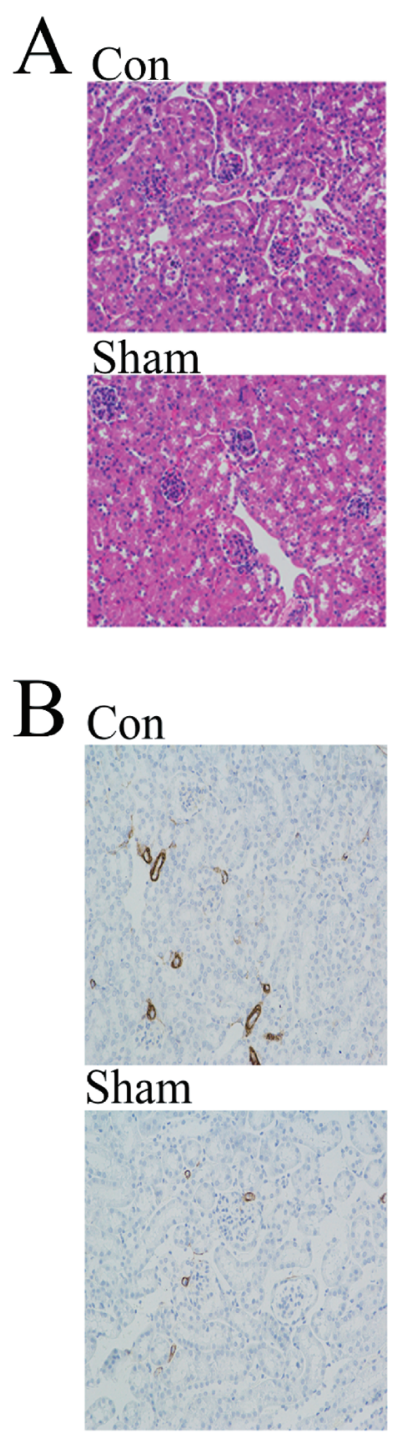
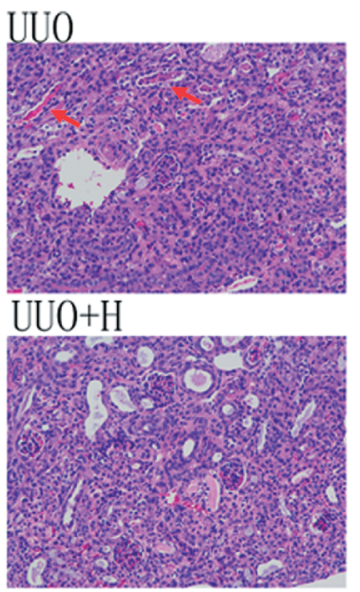

UU0

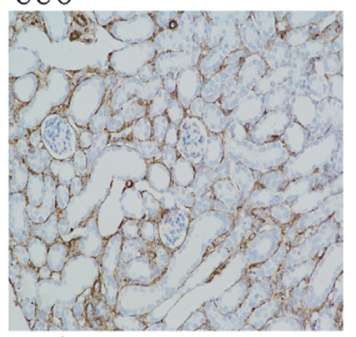

$\mathrm{UUO}+\mathrm{H}$

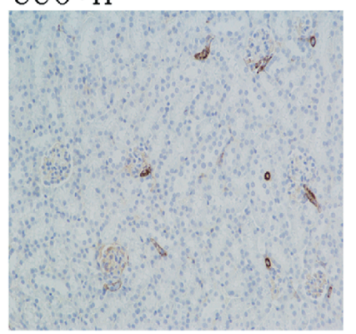

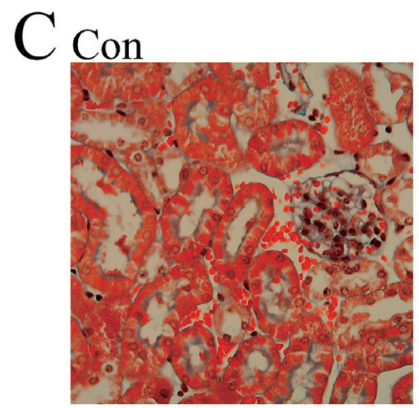

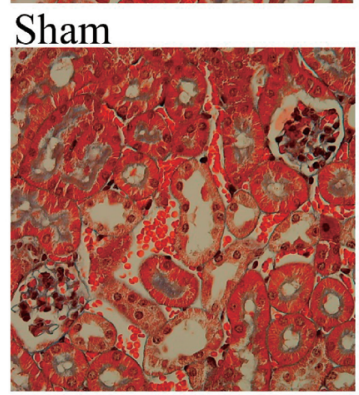

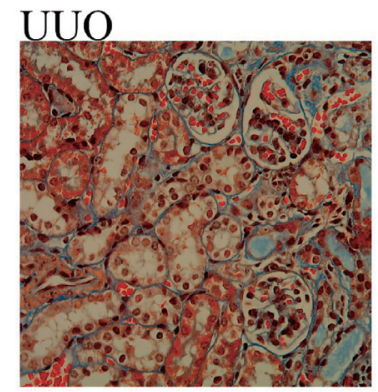

$\mathrm{UUO}+\mathrm{H}$

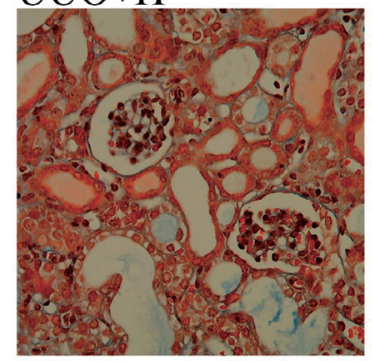

Fig. 1. The Treatment with HW Inhibited the Development of Interstitial Fibrosis in the Kidney

(A) Control group and Sham group, little change could be found (hematoxylin \& eosin $\times 200$ ). In UUO group, glomerular injury, base-membrane thickness and interstitial fibrosis could be observed along with protein cast (hematoxylin \& eosin $\times 200$ ). HW+UUO group, glomerular injury and interstitial fibrosis become slighter, more normal glomerulus appeared (hematoxylin $\&$ eosin $\times 200$ ). (B) Control group and Sham group, $\alpha$-SMA was mainly marked in capillary wall (Immunohistochemical staining $\times 200$ ). UUO group, $\alpha$-SMA distributed in renal interstitium (Immunohistochemical staining $\times 200$ ). HW + UUO group, $\alpha$-SMA was observed in glomerular basement membrane (Immunohistochemical staining $\times 200$ ). (C) Blue stain could rarely be found in renal interstitium in control group and Sham group $($ Masson $\times 400)$. In UUO group, renal interstitium was patently stained in blue (Masson $\times 400$ ). In HW+UUO group, blue stain alleviated remarkably in renal interstitium compared with UUO group in breadth and depth (Masson $\times 400$ ). Con, UUO, Sham and UUO+H represent control group, UUO group, Sham group and UUO+HW group respectively.

mild or severe glomerular injury along with protein cast (Fig. 1A) and increased the number of $\alpha$-SMA positive cells in renal interstitium (Fig. 1B) markedly in UUO group. After the treatment with HW, glomerular injury and tubular degeneration were observed mildly, and protein cast was rarely found (Fig. $1 \mathrm{~A}, \mathrm{UUO}+\mathrm{HW})$. In addition, $\alpha$-SMA positive myofibroblasts which produce collagen and participate in fibrotic diseased cells actively were significantly reduced to controllable levels after the treatment of HW in renal interstitium (Fig. 1B). Furthermore, in Masson staining, blue stain could rarely be found in renal interstitium in control group and sham group. In UUO group, renal interstitium was patently stained in blue. In HW+UUO group, blue stain alleviated remarkably in renal interstitium compared with UUO group in breadth and depth. The histologic examination has shown that the treatment with HW abolishes fibrosis induced by ureter ligation in kidney. Moreover, we discovered that UUO model induced up-regula- tion in $\alpha$-SMA, a myofibroblast marker, and down-regulation in epithelial marker E-cadherin (Figs. 2A, B).

HW Prevented EMT Induced by TGF- $\beta 1$ in HK-2 Cells HK-2 cells underwent a morphological change from epithelial to spindle mesenchymal-like phenotype (Fig. 3A) with TGF- $\beta 1(2.5 \mathrm{ng} / \mathrm{mL})$ for $36 \mathrm{~h}$, which simultaneously induced an up-regulation in $\alpha$-SMA and down-regulation in E-cadherin (Figs. 3B, C). The results are paralleled to the animal model. The HK-2 with EMT cells also express abundant fibronectin, an extracellular matrix component (Fig. 3D). The pretreatment with HW of HK-2 cells for $12 \mathrm{~h}$ abolished EMT induced by TGF- $\beta 1$ completely and the changes in $\alpha$-SMA, E-cadherin and fibronectin expression (Fig. 3).

HW Suppressed EMT Induced by TGF- $\beta 1$ in HK-2 Cells via the Regulation of Sirt1 Smad2, one of the downstream molecules of TGF- $\beta 1$, was up-regulated in HK-2 cells during EMT induced by TGF- $\beta 1$ (Fig. 4). The pretreatment with HW, 

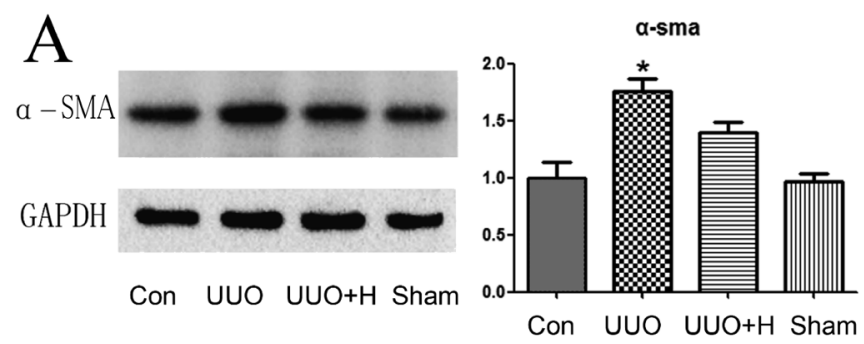

B

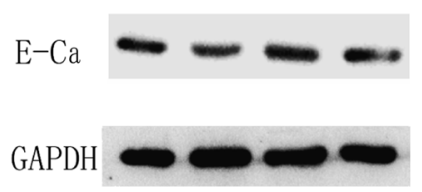

Con UUO UUO+H Sham

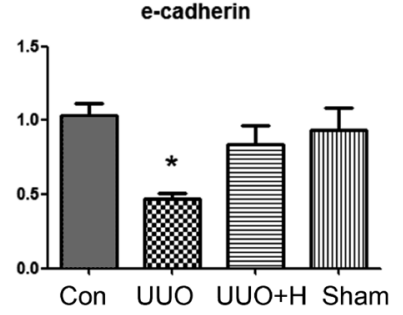

Fig. 2. The Treatment with HW Inhibited the Development of Interstitial Fibrosis in the Kidney

Western blot assay for $\alpha$-SMA and E-cadherin expressions in kidney tissue and graphical representation of the relative quantification for $\alpha$-SMA and E-cadherin The values of mean \pm S.E.M. $(n=3)$ were gained from relative abundance quantified by densitometry and normalized to GAPDH. ( $\alpha$-SMA: $* p<0.05 v$ s. Control group); (E-cadherin: $* p<0.05$ vs. TGF- $\beta 1$ group). One-way ANOVA followed by Tukey's multiple comparison test. Con, UUO, $\mathrm{UUO}+\mathrm{H}$ and Sham represent control group, UUO group, UUO+HW group and Sham group respectively.

A
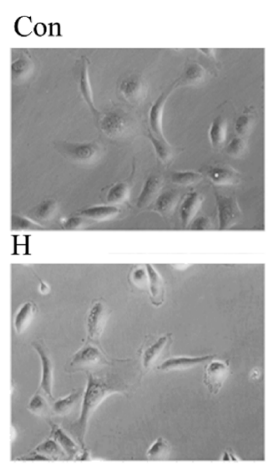

$\mathrm{E}$
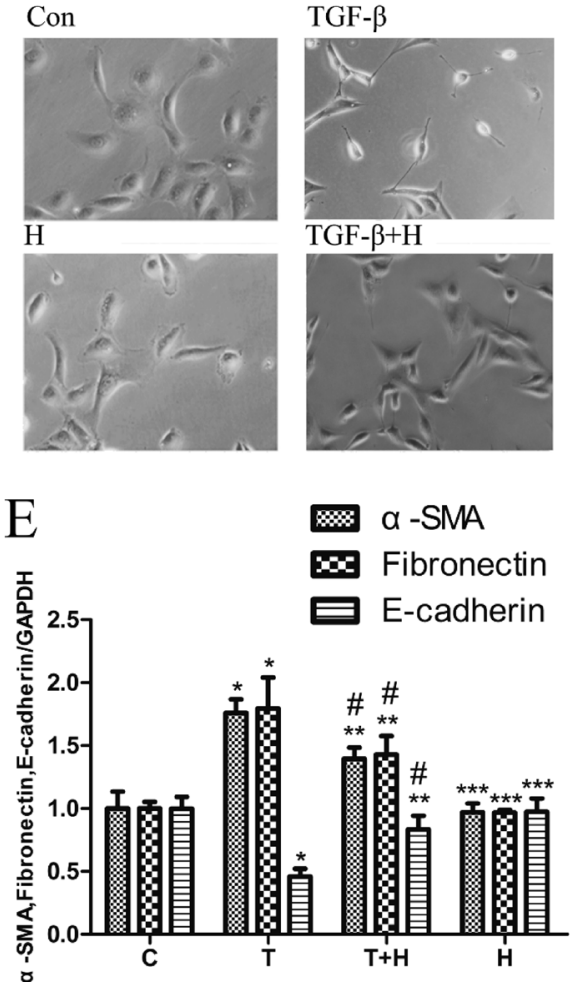

which abolished EMT, did not alter the expression of Smad2, implying that HW did not regulate TGF- $\beta 1$-Smad pathway to prevent fibrosis.

On the other side, Sirt1, anti-fibrotic molecule, was downregulated by TGF- $\beta 1$ in HK-2 cells during EMT, which was salvaged by the pretreatment with $\mathrm{HW}$ for $12 \mathrm{~h}$ (Fig. 5A). To further confirm the contribution of Sirtl to the effect of HW against EMT, Sirtinol, a pharmacological inhibitor of Sirt1, was employed to block the function of Sirt1. With treatment of HK-2 cells by Sirtinol at $20 \mu \mathrm{M}$ for $36 \mathrm{~h}$ to inhibit the activity of Sirt1, the effect of HW on E-cadherin in HK-2 cells was completely reversed (Fig. 5B), indicating Sirtl was the molecule to exert anti-fibrotic function of HW.

\section{DISCUSSION}

In the current study, we investigated the protective effect of hydrogen against renal fibrosis in UUO model and EMT induced by TGF- $\beta 1$ in HK- 2 cells, then we found that the treatment with HW inhibited EMT and the development of renal fibrosis, which was attributed to the expression of Sirt1 regulated by hydrogen.

EMT is a significant step towards fibrogenesis and it also presents the morphological changes associated with upregulation of fibronectin, $\alpha$-SMA and down-regulation of E-cadherin. ${ }^{10)}$ We find out that under the treatment with $\mathrm{HW}$,

B

$\mathrm{C}$

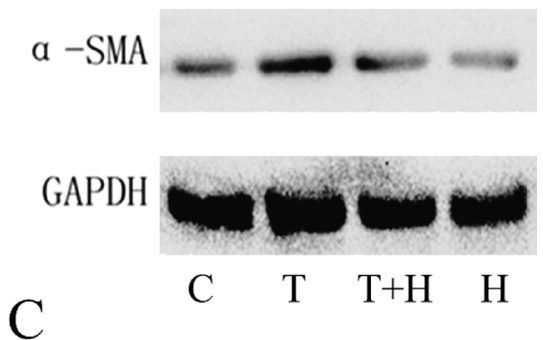

Fib
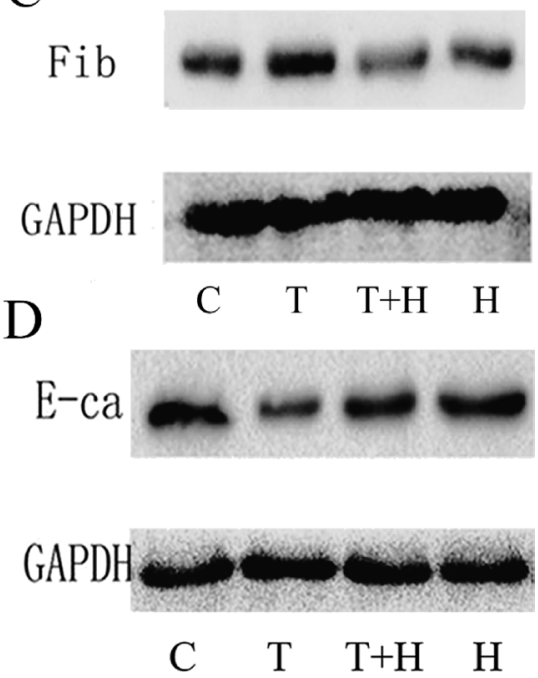

Fig. 3. HW Prevented EMT Induced by TGF- $\beta 1$ in HK-2 Cells

Pretreatment HW was given to HK-2 cells for $12 \mathrm{~h}$ and then TGF- $\beta 1$ was given to the cells for the following $36 \mathrm{~h}$. (A) The morphology of HK-2 cell was changed from normal shape to spindle-shape with TGF- $\beta 1$ stimulated compared with that of the control group, and the group with pretreatment of HW could reduce this change. (B-D) Western blot assay for fibronectin, E-cadherin and $\alpha$-SMA expressions in HK-2 cells and graphical representation of the relative quantification for fibronectin, E-cadherin and $\alpha$-SMA. (E) The relative values were calculated by the density of fibronectin, E-cadherin and $\alpha$-SMA $v s$. GAPDH (\%). The values of mean \pm S.E.M. ( $n=3)$ were gained from relative abundance quantified by densitometry and normalized to GAPDH. $\left(\alpha-\mathrm{SMA}: * p<0.05 v s\right.$. Control group, ${ }^{*} p<0.05$ vs. TGF- $\beta 1$ group, $* * p<0.05 v s$. Control group, ${ }^{* * *} p>0.05 v s$. Control group); (fibronectin : * $p<0.05 v s$. Control group, ${ }^{*} p<0.05$ vs. TGF- $\beta 1$ group, $* * p>0.05 v s$. Control group, $* * * p>0.05 v s$. Control group); (E-cadherin : ${ }^{\#} p<0.05 v s$. TGF- $\beta 1$ group, ${ }^{* *} p>0.05 v s$. Control group, ${ }^{* * *} p>0.05 v s$. Control group). One-way ANOVA followed by Tukey's multiple comparison test. $\mathrm{Con}(\mathrm{C})$, TGF- $\beta(\mathrm{T})$, TGF- $\beta+\mathrm{H}(\mathrm{T}+\mathrm{H})$ and $\mathrm{H}$ represent control group, TGF- $\beta 1$ group, TGF- $\beta 1+\mathrm{HW}$ group and HW group respectively. 


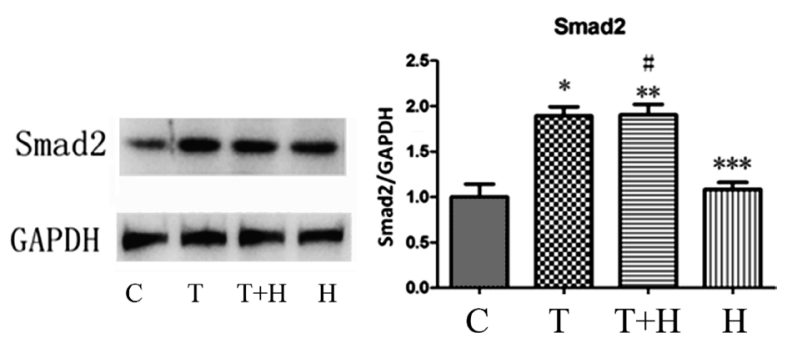

Fig. 4. HW Attenuated the Effect of EMT Which Had No Effect on Smad2

Western blot assay for the expressions of sirt1 in HK-2 cells and graphical representation of the relative quantification for $\operatorname{Smad} 2$. The values of mean \pm S.E.M $(n=3)$ were gained from relative abundance quantified by densitometry and normalized to GAPDH. $\left({ }^{*} p<0.01 v s\right.$. Control group, ${ }^{\#} p>0.05$ vs. TGF- $\beta 1$ group, ${ }^{* *} p<0.01$ $v s$. Control group, $* * * p>0.05 v s$. Control group). One-way ANOVA followed by Tukey's multiple comparison test. $\mathrm{C}, \mathrm{T}, \mathrm{T}+\mathrm{H}$ and $\mathrm{H}$ represent control group, TGF- $\beta 1$ group, TGF- $\beta 1+\mathrm{HW}$ group and HW group respectively.
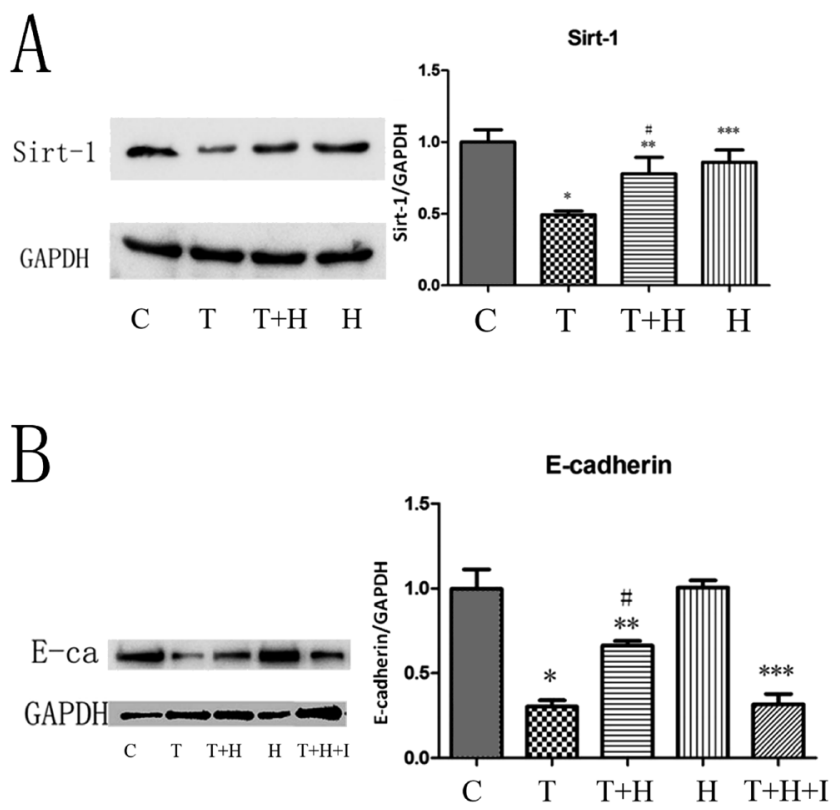

Fig. 5. The Anti-EMT Effect of HW on HK-2 Cells Was Blocked by the Inhibitors of Sirt1

(A) Western blot assay for the expressions of Sirt1 in HK-2 cells and graphical representation of the relative quantification for Sirt1. (B) Western blot assay for the expressions of E-cadherin in HK-2 cells and graphical representation of the relative quantification for E-cadherin. The relative values were calculated by the density of Sirtl and E-cadherin vs. GAPDH (\%). The values of mean \pm S.E.M. $(n=3)$ were gained from relative abundance quantified by densitometry and normalized to GAPDH. (A: ${ }^{*} p<0.05 v s$. Control group, ${ }^{\#} p<0.05 v s$. TGF- $\beta 1$ group, ${ }^{* *} p>0.05$ vs. Control group, $* * * p>0.05 v s$. Control group) (B: ${ }^{\#} p<0.01 v s$. HW+TGF- $\beta 1$ $* * * p>0.05$ vs. HW+TGF- $\beta 1$ ). One-way ANOVA followed by Tukey's multiple comparison test. $\mathrm{C}, \mathrm{T}, \mathrm{T}+\mathrm{H}, \mathrm{H}$ and $\mathrm{T}+\mathrm{H}+\mathrm{I}$ represent control group, TGF- $\beta 1$ group, TGF- $\beta 1+\mathrm{HW}$ group, HW group and TGF- $\beta 1+\mathrm{HW}+$ inhibitor group respectively.

the change of protein expression of $\alpha$-SMA, fibronectin and E-cadherin stimulated by TGF- $\beta 1$ was attenuated (Fig. 3). Besides, recent studies exhibited that HW could also attenuate the damage on retina and lung via up-regulating the expression of Sirt1. ${ }^{24)}$ Nevertheless, lack of attention was paid on the mechanism by which HW mediated the protection of renal fibrosis induced by TGF- $\beta 1$ or UUO. Thus we establish the anti-fibrotic role of HW in UUO model and renal tubular cell line.

In our study, we found out that HW exhibited the ability of improving renal function in UUO model as measured by BUN and Scr, and the ability of restoring renal injury and fibrosis as examined by histology and Western blot, which indicating that HW is able to inhibit the development of renal fibrosis and restore renal function (Figs. 1, 2).

TGF- $\beta 1$ is considered as a critical regulator of fibrosis, especially in the mechanism of fibrosis in vitro. ${ }^{25)} \mathrm{HK}-2$ cells were treated with TGF- $\beta 1$ to undergo EMT with significant up-regulation of $\alpha$-SMA, fibronectin and down-regulation of E-cadherin, indicating HK-2 cells lost the epithelial phenotype and transformed towards fibrotic morphology, while we found that HW could take a protective role of HK-2 cells (Fig. 3), which suggests that the preventive effect of $\mathrm{HW}$ on fibrosis could be mediated by depression of EMT. All of these data from our study indicate that HW prevents cell transformation towards fibroblast-like phenotype.

Smad proteins are downstream molecules of TGF- $\beta 1$ cell signaling pathway. To investigate the mechanism of renal fibrosis inhibited by HW, we tested Smad2 in EMT induced by TGF- $\beta 1$ in HK- 2 cells and found that HW showed no effect on TGF- $\beta 1$-induced increase in Smad2 expression (Fig. 4), which prompted us to seek new regulatory mechanism of HW.

In recent years, some studies have showed that the inhibition or inactivation of Sirtl can enhance the fibrosis following renal injury. ${ }^{26,27)}$ In our study, HW itself did not regulate the expression of Sirt1, however, HW abolished down-regulation of Sirt1 induced by TGF- $\beta 1$ (Fig. 5A). Moreover, when Sirt1 was inhibited by sirtinol, a Sirtl inhibitor, HW failed intervention with EMT induced by TGF- $\beta 1$ (Fig. 5B). Further studies on how HW regulates TGF- $\beta 1$ and Sirtl pathway are under investigation.

In conclusion, our study reveals that HW is able to alleviate the renal fibrosis induced by UUO in animals, and prevent the HK-2 cells from transforming to EMT induced by TGF- $\beta 1$, in which Sirt1 other than TGF- $\beta 1 / \mathrm{Smad} 2$ pathway is involved. The finding from our study implies a therapeutic potential of hydrogen against fibrosis, and Sirtl may be a latent target of the treatment and diagnosis of fibrosis.

Acknowledgments This project is financially supported by the Medical Science and Technique Foundation of Jiangsu Province, P. R. China (No. BL2012045).

Conflict of Interest The authors declare no conflict of interest.

\section{REFERENCES}

1) Collins AJ, Foley RN, Chavers B, Gilbertson D, Herzog C, Johansen K, Kasiske B, Kutner N, Liu J, St Peter W, Guo H, Gustafson S, Heubner B, Lamb K, Li S, Li S, Peng Y, Qiu Y, Roberts T, Skeans M, Snyder J, Solid C, Thompson B, Wang C, Weinhandl E, Zaun D, Arko C, Chen SC, Daniels F, Ebben J, Frazier E, Hanzlik C, Johnson R, Sheets D, Wang X, Forrest B, Constantini E, Everson S, Eggers P, Agodoa L. United States Renal Data System 2011 Annual Data Report: Atlas of chronic kidney disease \&amp; end-stage renal disease in the United States. Am. J. Kidney Dis., 59 (A7), e1-e420 (2012).

2) Solez K, Colvin RB, Racusen LC, Sis B, Halloran PF, Birk PE, Campbell PM, Cascalho M, Collins AB, Demetris AJ, Drachenberg CB, Gibson IW, Grimm PC, Haas M, Lerut E, Liapis H, Mannon RB, Marcus PB, Mengel M, Mihatsch MJ, Nankivell BJ, Nickeleit V, Papadimitriou JC, Platt JL, Randhawa P, Roberts I, SalinasMadriga L, Salomon DR, Seron D, Sheaff M, Weening JJ. Banff '05 Meeting Report: differential diagnosis of chronic allograft injury 
and elimination of chronic allograft nephropathy ('CAN'). Am. J. Transplant., 7, 518-526 (2007).

3) Xin HG, Zhang BB, Wu ZQ, Hang XF, Xu WF, Ni W, Zhang RQ, Miao XH. Consumption of hydrogen-rich water alleviates renal injury in spontaneous hypertensive rats. Mol. Cell. Biochem., 392, $117-124$ (2014)

4) Katakura M, Hashimoto M, Tanabe Y, Shido O. Hydrogen-rich water inhibits glucose and alpha,beta-dicarbonyl compound-induced reactive oxygen species production in the SHR.Cg-Leprcp/NDmcr rat kidney. Med. Gas Res., 2, 18 (2012).

5) Abe T, Li XK, Yazawa K, Hatayama N, Xie L, Sato B, Kakuta Y, Tsutahara K, Okumi M, Tsuda H, Kaimori JY, Isaka Y, Natori M, Takahara S, Nonomura N. Hydrogen-rich University of Wisconsin solution attenuates renal cold ischemia-reperfusion injury. Transplantation, 94, 14-21 (2012).

6) Li FY, Zhu S, Wang Z, Wang H, Zhao Y, Chen G. Consumption of hydrogen-rich water protects against ferric nitrilotriacetate-induced nephrotoxicity and early tumor promotional events in rats. Food Chem. Toxicol., 61, 248-254 (2013).

7) Matsushita T, Kusakabe Y, Kitamura A, Okada S, Murase K. Protective effect of hydrogen-rich water against gentamicin-induced nephrotoxicity in rats using blood oxygenation level-dependent MR imaging. Magn. Reson. Med. Sci., 10, 169-176 (2011).

8) Kitamura A, Kobayashi S, Matsushita T, Fujinawa H, Murase K. Experimental verification of protective effect of hydrogen-rich water against cisplatin-induced nephrotoxicity in rats using dynamic contrast-enhanced CT. Br. J. Radiol., 83, 509-514 (2010).

9) Nakashima-Kamimura N, Mori T, Ohsawa I, Asoh S, Ohta S. Molecular hydrogen alleviates nephrotoxicity induced by an anticancer drug cisplatin without compromising anti-tumor activity in mice. Cancer Chemother. Pharmacol., 64, 753-761 (2009).

10) Guo L, Peng W, Tao J, Lan Z, Hei H, Tian L, Pan W, Wang L, Zhang X. Hydrogen sulfide inhibits transforming growth factorbetal-induced EMT via Wnt/Catenin pathway. PLOS ONE, 11, e0147018 (2016).

11) Jimenez-Sousa MA, Fernandez-Rodriguez A, Heredia M, Tamayo E, Guzman-Fulgencio M, Lajo C, Lopez E, Gomez-Herreras JI, Bustamante J, Bermejo-Martin JF, Resino S. Genetic polymorphisms located in TGFB1, AGTR1, and VEGFA genes are associated to chronic renal allograft dysfunction. Cytokine, 58, 321-326 (2012).

12) Tikoo K, Tripathi DN, Kabra DG, Sharma V, Gaikwad AB. Intermittent fasting prevents the progression of type I diabetic nephropathy in rats and changes the expression of Sir2 and p53. FEBS Lett., 581, 1071-1078 (2007).

13) Chuang PY, Dai Y, Liu R, He H, Kretzler M, Jim B, Cohen CD, He JC. Alteration of forkhead box $\mathrm{O}$ (foxo4) acetylation mediates apoptosis of podocytes in diabetes mellitus. PLOS ONE, 6, e23566 (2011).

14) Huang K, Huang J, Xie X, Wang S, Chen C, Shen X, Liu P, Huang $\mathrm{H}$. Sirtl resists advanced glycation end products-induced expressions of fibronectin and TGF-betal by activating the Nrf2/ARE pathway in glomerular mesangial cells. Free Radic. Biol. Med., 65,
528-540 (2013).

15) Hayashida K, Sano M, Ohsawa I, Shinmura K, Tamaki K, Kimura K, Endo J, Katayama T, Kawamura A, Kohsaka S, Makino S, Ohta S, Ogawa S, Fukuda K. Inhalation of hydrogen gas reduces infarct size in the rat model of myocardial ischemia-reperfusion injury. Biochem. Biophys. Res. Commun., 373, 30-35 (2008).

16) Lin Z, Yang H, Kong Q, Li J, Lee SM, Gao B, Dong H, Wei J, Song J, Zhang DD, Fang D. USP22 antagonizes p53 transcriptional activation by deubiquitinating Sirt1 to suppress cell apoptosis and is required for mouse embryonic development. Mol. Cell, 46, 484-494 (2012)

17) Frye RA. Phylogenetic classification of prokaryotic and eukaryotic Sir2-like proteins. Biochem. Biophys. Res. Commun., 273, 793-798 (2000).

18) Chen $\mathrm{D}$, Steele AD, Lindquist $\mathrm{S}$, Guarente L. Increase in activity during calorie restriction requires Sirt1. Science, 310, 1641 (2005).

19) Boily G, Seifert EL, Bevilacqua L, He XH, Sabourin G, Estey C, Moffat C, Crawford S, Saliba S, Jardine K, Xuan J, Evans M, Harper ME, McBurney MW. SirT1 regulates energy metabolism and response to caloric restriction in mice. PLoS ONE, 3, e1759 (2008).

20) Vinciguerra M, Santini MP, Martinez C, Pazienza V, Claycomb WC, Giuliani A, Rosenthal N. mIGF-1/JNK1/SirT1 signaling confers protection against oxidative stress in the heart. Aging Cell, 11, 139-149 (2012).

21) Cai J, Kang Z, Liu K, Liu W, Li R, Zhang JH, Luo X, Sun X. Neuroprotective effects of hydrogen saline in neonatal hypoxia-ischemia rat model. Brain Res., 1256, 129-137 (2009).

22) Djudjaj S, Chatziantoniou C, Raffetseder U, Guerrot D, Dussaule JC, Boor P, Kerroch M, Hanssen L, Brandt S, Dittrich A, Ostendorf T, Floege J, Zhu C, Lindenmeyer M, Cohen CD, Mertens PR. Notch-3 receptor activation drives inflammation and fibrosis following tubulointerstitial kidney injury. J. Pathol., 228, 286-299 (2012).

23) Zecher M, Guichard C, Velasquez MJ, Figueroa G, Rodrigo R. Implications of oxidative stress in the pathophysiology of obstructive uropathy. Urol. Res., 37, 19-26 (2009).

24) Qi LS, Yao L, Liu W, Duan WX, Wang B, Zhang L, Zhang ZM. Sirtuin type 1 mediates the retinal protective effect of hydrogenrich saline against light-induced damage in rats. Invest. Ophthalmol. Vis. Sci., 56, 8268-8279 (2015).

25) Shull MM, Ormsby I, Kier AB, Pawlowski S, Diebold RJ, Yin M, Allen R, Sidman C, Proetzel G, Calvin D, Annunziata N, Doetschman T. Targeted disruption of the mouse transforming growth factor-beta 1 gene results in multifocal inflammatory disease. Nature, 359, 693-699 (1992).

26) Kida Y, Zullo JA, Goligorsky MS. Endothelial sirtuin 1 inactivation enhances capillary rarefaction and fibrosis following kidney injury through Notch activation. Biochem. Biophys. Res. Commun., 478, 1074-1079 (2016)

27) Vasko R, Xavier S, Chen J, Lin CH, Ratliff B, Rabadi M, Maizel J, Tanokuchi R, Zhang F, Cao J, Goligorsky MS. Endothelial sirtuin 1 deficiency perpetrates nephrosclerosis through downregulation of matrix metalloproteinase-14: relevance to fibrosis of vascular senescence. J. Am. Soc. Nephrol., 25, 276-291 (2014). 